\title{
ON THE ALGEBRA OF REPRESENTATIVE FUNCTIONS OF A LIE ALGEBRA
}

\author{
BY \\ WILLIAM B. GILES
}

1. Introduction. This paper deals with the structure of the algebra of representative functions for Lie algebras. We shall be concerned mostly with proving analogs of certain of the results which are known from [5] for the case of Lie groups. Our results may also be viewed as a natural extension of [2] and [3].

More specifially, in $\S \S 3$ and 4, we treat a special class of subalgebras of $R(L)$, the algebra of representative functions on the universal enveloping algebra of a Lie algebra $L$, called normal basic subalgebras, which are fundamental for the representation theory. For example, we prove that the "semisimple part" of such a subalgebra is always the same, and prove a conjugacy theorem for these subalgebras.

In $\S 5$, we turn our attention to the group of the proper automorphisms of $R(L)$. We analyze its intrinsic structure, and also obtain this group as the inverse limit of its restriction images on the finite-dimensional stable subspaces of $R(L)$. These considerations lead naturally to the discussion of a more general inverse limit system, namely of irreducible algebraic linear groups and rational group epimorphisms, which we undertake in $\$ 6$.

In conclusion, the auther wishes to thank Professor Hochschild for his invaluable assistance in the writing of this thesis.

2. Notations and terminology. In this section, we recall some terminology and results, mostly from [2] and [3], and introduce some notation which will be standard throughout this paper. However, we do not attempt to make our work self-contained.

$F$ will always be a field of characteristic zero. All tensor products will be taken over $F$. If $V$ is an $F$-space, $V^{\prime}$ will denote its dual and $E_{v}$ will denote the algebra $\operatorname{Hom}_{F}(V, V)$. $L$ will be a finite-dimensional Lie algebra over $F, A$ its radical, $N=[L, A]$ its nilpotent radical, and $H$ a fixed maximal semisimple subalgebra of $L$. The representations we consider will be finite-dimensional. $U(L)$ (or just $U$ ) will denote the universal enveloping algebra of $L$. A representation of $L$ on $V$ is simultaneously considered as a unitary left $U(L)$-module structure on $V$.

If $(\rho, V)$ is a representation of $L$, an $F$-valued function on $U(L)$ of the form $\lambda \circ \rho$ with $\lambda \in E(V)^{\prime}$ is called a representative function associated with $\rho$. The set

Received by the editors September 25, 1962. 
of all representative functions associated with all representations of $L$ clearly forms an $F$-space, which is called the space of representative functions on $U(L)$, and is denoted by $R(L)$ (or by $R$ ).

The unitary homomorphism $d: U(L) \rightarrow U(L) \otimes U(L)$ which is characterized by $d(x)=x \otimes 1+1 \otimes x$ for each $x \in L$, gives by dualizing a commutative associative algebra structure on $U(L)^{\prime}$. For $f, g \in U(L)^{\prime}$ their product is denoted by $f g$. If $f$ and $g$ actually belong to $R(L)$, it is easily shown that $f g$ belongs to $R(L)$. Hence there is an induced algebra structure on $R(L)$. The space of representative functions, with this algebra structure, is called the algebra of representative functions on $U(L)$, and is still denoted by $R(L)$.

For $f \in U(L)^{\prime}$ and $u \in U(L)$, the left translate of $f$ by $u, u \cdot f$, is defined by $(u \cdot f)(v)=f(v u)$ for each $v \in U(L) . U(L)^{\prime}$ becomes a left $U(L)$-module under left translation. If $f \in R(L)$, then $U(L) \cdot f \subset R(L)$. The elements of $R(L)$ are precisely those $f \in U(L)^{\prime}$ such that $U(L) \cdot f$ has finite $F$-dimension. Analogous results hold for $f \cdot u$, the right translate of $f$ by $u$, which is defined by $(f \cdot u)(v)=f(u v)$.

An element $f \in R(L)$ is said to be semisimple if the representation of $L$ by left translations on $U(L) \cdot f$ is semisimple. All representative functions which are associated with semisimple representations are semisimple. The set of semisimple representative functions on $U(L)$ forms a subalgebra of $R(L)$, denoted by $R(L)_{s}$. For any subalgebra $T \subset R(L)$ and any subalgebra $K$ of $L, T^{K}$ will denote the set of those elements $f \in T$ for which $K \cdot f=\{0\} . T \cap R(L)_{s}$ will be called the semisimple part of $T$ and denoted by $T_{s}$.

A basis $x_{1}, \cdots, x_{m}$ may be introduced in $N$ such that, for $1 \leqq i, j \leqq n,\left[x_{i}, x_{j}\right]$ is a linear combination of the $x_{k}$ 's with $k<\min (i, j) . x_{1}, \cdots, x_{m}$ may then be completed to a basis $x_{1}, \cdots, x_{n}$ for $A$ in such a way that the elements $x_{m+1}, \cdots, x_{n}$ belong to a nilpotent subalgebra $T$ of $A$ such that $[T, H]=\{0\}$. The elements of $U(L)$ may be represented uniquely as sums of monomials $u x_{n}^{e_{n}} \cdots x_{1}^{e_{1}}$ with $u \in U(H)$. Denote by $u_{0}$ the component of $u$ in $F$ in the standard decomposition $U(H)$ $=F+H U(H)$.

Define functions $g_{i} \in R(L)$ by

$$
g_{i}\left(u x_{n}^{e_{n}} \cdots x_{1}^{e_{1}}\right)=u_{0} \delta_{0 e_{n}} \cdots \delta_{1 e_{i}} \cdots \delta_{0 e_{1}}, \quad i=1, \cdots, n,
$$

where the $\delta_{j k}$ are the Kronecker functions. The $F$-linear combinations of the $g_{m+1}, \cdots, g_{n}$ are called the elementary functions and denoted by $E$. They are precisely those representative functions which vanish on $F$ and $L^{2} U(L)$. A representative function $f$ is said to be a constant function if $f(L U(L))=\{0\}$, in which case $f$ is identified with $f(1) \in F$. The subalgebra $F\left[g_{1}, \cdots, g_{n}\right]$ of $R(L)$ is denoted by $V$. It is stable under left translation.

For each elementary function $f$ and each $c \in F^{*}$, the algebraic closure of $F$, the function $\exp (c f)$ is well defined as a function from $U(L)$ to $F^{*}$. Those $F^{*}$ - 
linear combinations of the $\exp (c f)$ which actually take values in $F$ are called the trigonometric functions on $U(L)$ and constitute a subalgebra $C$ of $R(L)$. Let $Q$ denote the multiplicative group of the unitary homomorphisms of $U(L)$ into $F$. In case $F$ is algebraically closed, the elements of $Q$ form an $F$-basis for $C$. Denote the subalgebra $R(L)^{A} V$ of $R(L)$ by $B$. Then $B$ is stable under the left translations and $R(L)=B \otimes C$.

If $T$ is a subalgebra of $R(L)$ that is stable under the right translations and contains the constant functions, then an automorphism of $T$ is said to be proper if it commutes with the right translations and leaves the constants fixed.

3. Basic subalgebras of $R(L)$. We begin with two well-known lemmas, which are reproduced here for completeness.

LEMMA 3.1. Let $I$ be an ideal of $L$ and let $V$ be a semisimple representation space for $L$. Then the induced representation of $I$ on $V$ is semisimple.

Proof. The induced representation of $I$ is semisimple if and only if the induced representation of $I+N$ is semisimple. Hence, we may assume that $I \supset N$. The representation of $L$ on $V$ induces a semisimple representation of $L / N$ on $V$. The representation of $I / N$ induced from this representation of $L / N$ is semisimple if and only if the representation of $I$ induced from the representation of $L$ is semisimple. Hence, we may assume that $L=H \oplus A$, with $H$ semisimple and $A$ abelian.

The induced representation of $A$ on $V$ is semisimple since $A$ is a direct summand of $L$. Also, $I=H^{\prime} \oplus A^{\prime}$, where $A^{\prime}=I \cap A$ and $H^{\prime}=I \cap H$. As an ideal of $H$, $H^{\prime}$ is semisimple. Hence, the induced representations of $H^{\prime}$ and $A^{\prime}$ on $V$ are semisimple, which implies that the induced representation of $I$ is semisimple. q.e.d

LEMMA 3.2. Let $I$ be an ideal of $L$ such that $L / I$ is semisimple. Let $V$ be a representation space for $L$ and suppose that $V$ is semisimple for its induced structure as a representation space for $I$. Then $V$ is semisimple as a representation space for $L$.

Proof. Since $L / I$ is semisimple, we have $I \supset A$. Hence, if the conditions of the lemma hold for $I$, they also hold for $A$, by the previous lemma, and so it suffices to prove the lemma for $I=A$.

The induced representation of $N$ is nilpotent. Since $N$ is an ideal of $A$ and the induced representation of $A$ is assumed semisimple, the induced representation of $N$ is also semisimple, again by the previous lemma. Hence, the induced representation of $N$ is zero. Finally, the induced representation of $H \oplus A / N$, i.e., of $L / N$, is semisimple, whence the original representation of $L$ is semisimple.

Next we introduce a class of subalgebras of $R(L)$ which will be of importance in the sequel. 
Definitions. A subalgebra $D$ of $R(L)$ is called a basic subalgebra if

(1) $D$ contains the constant functions,

(2) $R(L)=D \otimes C$.

A basic subalgebra $D$ of $R(L)$ is called a normal basic subalgebra if $D$ is stable under left translations, and if $D_{s}$, the semisimple part of $D$, is stable under both right and left translations.

For example, in [3], the subalgebra $B$ described in $\S 2$ above was shown to be a basic subalgebra invariant under left translations. By [3, p. 614], any left stable basic subalgebra necessarily contains $E$.

Lemma 3.3. Assume that $F$ is algebraically closed and let $D$ be a normal basic subalgebra of $R(L)$. Then $R_{s}=D_{s}[Q]$.

Proof. It is clear that $R_{s} \supset D_{s}[Q]$. Suppose that $f \in R_{s}$. Then $f$ may be written uniquely in the form $f=\Sigma_{q \in Q} d_{q}(f) q$ with each $d_{q}(f) \in D$. Let $x \in L$. Then

$$
x \cdot f=\Sigma_{q \in Q}\left(x \cdot d_{q}(f)+q(x) d_{q}(f)\right) q .
$$

It follows that $U(L) \cdot d_{q}(f) q$ is a $U(L)$-homomorphic image of $U(L) \cdot f$, for each $q \in Q$, and so is semisimple as a $U(L)$-module. Hence, each $d_{q}(f) q$ is a semisimple representative function. But each $q \in Q$ is invertible and its inverse is semisimple. Therefore, for each $q \in Q,\left(d_{q}(f) q\right) q^{-1}=d_{q}(f)$ is semisimple. Hence $R_{s}=D_{s}[Q]$. q.e.d.

LEMMA 3.4. $B_{s}=R^{A}$ and so $B$ is a normal basic subalgebra of $R(L)$. Under the representation of $L$ by left translations on $R(L)$, the annihilator of $B_{s}$ is $A$.

Proof. Clearly, $R^{A} \subset B_{s}$. Suppose that $f \in B_{s}$. By Lemma 3.1, $U(L) \cdot f$ is semisimple as a $U(A)$-module. We may write $f=\sum_{i=1}^{t} r_{i} v_{i}$ with $r_{i} \in R^{A}$ and $v_{i} \in V$ for each $i$. Futhermore, we may choose the $r_{i}$ to be linearly independent over $F$, in which case the $v_{i}$ are uniquely determined by the $r_{i}$ and $f$. Let $x \in A$. Then $x \cdot f=\sum_{i=1}^{t} r_{i}\left(x \cdot v_{i}\right)$. Hence, for each $i, U(A) \cdot v_{i}$ is a $U(A)$-homomorphic image of $U(A) \cdot f$ and therefore is semisimple as a $U(A)$-module.

Since $U(L)=U(A) U(H)$, it follows that, for each $i$,

$$
U(L) \cdot v_{i}=U(A) U(H) \cdot v_{i}=\sum_{h \in U(H)} U(A)\left(h \cdot v_{i}\right) .
$$

We assert that, for arbitrary $h \in U(H)$, there exists a $U(A)$-epimorphism $U(A) \cdot v_{i} \rightarrow U(A)\left(h \cdot v_{i}\right)$ sending each $a \cdot v_{i}$ onto $a h \cdot v_{i}$. It is sufficient to prove this for elements $h$ that are monomials with respect to some canonical basis of $U(H)$. First, we note that, since $a \cdot f \rightarrow a \cdot v_{i}$ is a $U(A)$-epimorphism of $U(A) \cdot f$ onto $U(A) \cdot v_{i}$ and $f$ is semisimple, $N \cdot v_{i}=\{0\} . N$ being an ideal in $L, R^{N}$ is twosidedly stable, and so, in particular, $[H, U(A)] U(H) \cdot v_{i}=\{0\}$.

To prove the assertion, it suffices to show that, for $a \in U(A), a \cdot v_{i}=0$ implies $a h \cdot v_{i}=0$. This can be done by induction on the filtration index of $h$ in the usual 
filtration of $U(H)$. For $h$ of filtration index zero, the result is clear, so assume that the filtration index of $h$ is greater than zero and that the result has been proved for all monomial elements of lower filtration index than $h$. Write $h=x h^{\prime}$ with $x \in H$ and $h^{\prime}$ a monomial of lower filtration index than $h$. Then $a \cdot v_{i}=0$ gives

as required.

$$
a h \cdot v_{i}=a x h^{\prime} \cdot v_{i}=-x a h^{\prime} \cdot v_{i}-[x, a] h^{\prime} \cdot v_{i}=0
$$

This shows that $U(A)\left(h \cdot v_{i}\right)$ is semisimple as a $U(A)$-module for each $h \in U(H)$, and so, by (1), that $U(L) \cdot v_{i}$ is semisimple as a $U(A)$-module. Therefore, by Lemma 3.2, $U(L) \cdot v_{i}$ is semisimple as a $U(L)$-module. Hence, each $v_{i}$ lies in $R_{s}$, and so to complete the proof of the first part of the proposition, it suffices to show that $V \cap R_{s}$ consists only of the constant functions. For this, assume $g \in V \cap R_{s}$ and write $g$ as a polynomial in the $g_{i}$ with coefficients in $F$. Since $g \in R_{s}$, we have $N \cdot g=\{0\}$ and so $g$ is actually a polynomial in the $g_{i}$ with $i \geqq m$. But then if $k$ is the total degree of this polynomial, $L^{k+1} \cdot g=\{0\}$ and so the representation of $L$ by left translations on $U(L) \cdot g$ is both semisimple and nilpotent; that is, it is trivial. Hence, $g$ is a constant.

To show that $B$ is normal, we need only note that $R^{A}$ is two-sidedly stable. By definition, the annihilator of $R^{A}$ contains $A . R^{A}$ is naturally isomorphic with $R(L / A)$ and the canonical image of the annihilator of $R^{A}$ in $L / A$ is the annihilator of $R(L / A)$, i.e., is zero. Therefore, the annihilator of $R^{A}$ is precisely $A$. This completes the proof.

THEOREM 3.1. Let $L$ be a Lie algebra over a field $F$ of characteristic zero. Let $R$ be the algebra of representative functions on $L$ and let $A$ be the radical of $L$. If $D$ is any normal basic subalgebra of $R$, then $D_{s}=R^{A}$.

Proof. Assume first of all that $F$ is algebraically closed. We note that [5, p. 113, Proposition 3.1] applies equally well in our case, the proof being as in [5], with appropriate reinterpretation. If $\phi$ is the coefficient sum homomorphism from $B$ to $D$, then $\phi^{-1}$ is the coefficient sum homomorphism from $D$ to $B$. From Lemma 3.3 , it follows that $\phi\left(B_{s}\right) \subset D_{s}$ and $\phi^{-1}\left(D_{s}\right) \subset B_{s}$. Thus $\phi$ maps $B_{s}$ isomorphically onto $D_{s}$.

For $x \in L$, we define a map $\phi_{x}: B_{s} \rightarrow F$ by $\phi_{x}(f)=\phi(f)(x)$. Then $\phi_{x}$ is a differentiation of $B_{s}=R^{A}$. By [1, p. 502, Proposition 1], $\phi_{x}$ defines a unique proper derivation of $B_{s}$ such that $\zeta(f)(1)=\phi_{x}(f)$ for each $f \in B_{s}$. $B_{s}$ may be identified with the algebra of all representative functions on the semisimple Lie algebra $H$. It follows then from [2, p. 612, Theorem 2] that $\zeta$ is the left translation by an element $x_{1} \in H$, so that we have $\phi(f)(x)=f\left(x_{1}\right)$ for each $f \in B_{s}$.

Now let $d \in D_{s}$ and write $d=\Sigma_{q \in Q} b_{q}(d) q$ with $b_{q}(d) \in B_{s}$. Then $d\left(x_{1}\right)=\Sigma_{q \in Q} b_{q}(d)\left(x_{1}\right)$ since $q\left(x_{1}\right)=0$ for each $q \in Q$, and hence $d\left(x_{1}\right)=\phi\left(\Sigma_{q \in Q} b_{q}(d)\right)(x)$. Since $\phi^{-1}$ is the coefficient sum homomorphism from $D$ to $B$, we have $\phi\left(\Sigma_{q \in Q} b_{q}(d)\right)=d$. Hence, $d\left(x_{1}\right)=d(x)$. Since $D$ is a normal basic 
basic subalgebra, $D_{s}$ is stable under the right translations. We conclude that $(d \cdot y)\left(x_{1}\right)=(d \cdot y)(x)$ for every $d \in D_{s}$ and $y \in L$. This gives $x_{1} \cdot d=x \cdot d$ for every $d \in D_{s}$. Let $K$ denote the kernel of the representation of $L$ by left translations on $D_{s}$. The last result shows that $L=H+K$. The mapping $\phi: B_{s} \rightarrow D_{s}$ is a $U(H)$-module isomorphism. Therefore, since the representation of $H$ by left translations on $B_{s}$ is faithful, we see that the representation of $H$ by left translations on $D_{s}$ is faithful. That is, $H \cap K=\{0\}$, and $L$ is a semi direct sum $H+K$. We have $N \subset K$ and $K / N \approx L /(H+N) \approx A / N$ and so $K$ is solvable. Hence $K=A$ and so $D_{s} \subset B_{s}$. We recall that $R_{s}=D_{s}[Q]=B_{s}[Q]$ and that the elements of $Q$ are free over $B_{s}$. This gives $D_{s}=B_{s}$ and so the theorem is proved for an algebraically closed field.

In case $F$ is not necessarily algebraically closed, let $F^{*}$ denote the algebraic closure of $F$. Then there are natural isomorphisms

$$
R\left(L \otimes F^{*}\right) \approx R(L) \otimes F^{*} \approx\left(C \otimes F^{*}\right) \otimes\left(D \otimes F^{*}\right)
$$

by means of which we identify these algebras. Then $C \otimes F^{*}$ is precisely the algebra of the trigonometric functions on $L \otimes F^{*}$, and the above shows that $D \otimes F^{*}$ is a normal basic subalgebra of $R\left(L \otimes F^{*}\right)$. We recall that semisimplicity is preserved under extension or restriction of scalars and that the radical of $L \otimes F^{*}$ is $A \otimes F^{*}$. Hence,

$$
D_{s} \otimes F^{*}=\left(D \otimes F^{*}\right)_{s}=\left(R \otimes F^{*}\right)^{A \otimes F^{*}}=R^{A} \otimes F^{*}
$$

and so $D_{s}=R^{A}$ and the theorem is proved.

COROLlary 3.1. If $L$ is solvable, then every basic subalgebra of $R$ that is stable under the left translations is a normal basic subalgebra. Hence, its semisimple part consists of the constant functions.

Proof. If $L$ is solvable, then $N=[L, L]$, whence $L / N$ is abelian. Let $D$ be a left stable basic subalgebra of $R$. The elements of $D_{s}$ are annihilated by left translations with elements of $N$. Hence, for each $x \in L$ and each $f \in D_{s}$ we have $f \cdot x=x \cdot f$, which proves two-sided stability.

4. Conjugacy of normal basic subalgebras. We denote by $u \rightarrow u^{*}$ the algebra anti-automorphism of $U(L)$ that is characterized by $x^{*}=-x$ for each $x \in L$. The map $u \rightarrow u^{*}$ induces an automorphism $f \rightarrow f^{*}$ of $R(L)$ given by $f^{*}(u)=f\left(u^{*}\right)$ for each $x \in U(L)$.

We have the following lemma, the first statement of which is the right analog of [2, p. 501, Lemma 1$]$.

LEMMA 4.1. Let $T$ be a subspace of $R(L)$ that is stable under the left translations and let $\alpha$ be a linear map of $T$ into $U(L)^{\prime}$ which commutes with the left translations. Then every subspace of $T$ which is stable under the right translations is also stable under $\alpha$. 
Suppose that $T$ is actually a two-sidedly stable subalgebra of $R(L)$ containing the constants and stable under the involution $f \rightarrow f^{*}$, and that $\alpha$ is also multiplicative and leaves the constants fixed. Then $\alpha$ is necessarily an automorphism of $T$ and the inverse of $\alpha$ is given by

$$
\alpha^{-1}(f)(x)=\alpha\left((x \cdot f)^{*}\right)(1) .
$$

Proof. Suppose $T$ and $\alpha$ are as in the first statement of the lemma. Let $f \in T$ and choose a basis $h_{1}, \cdots, h_{n}$ for the space spanned by the right translates of $f$. Using a general lemma about linearly independent maps of an arbitrary set into a field, we may choose elements $x_{1}, \cdots, x_{n}$ in $U(L)$ such that $h_{i}\left(x_{j}\right)=\delta_{i j}$ for each $i$ and $j$. This gives $f \cdot x=\sum_{i=1}^{n}\left(x_{i} \cdot f\right)(x) h_{i}$ for each $x \in U(L)$ and so

$$
x \cdot f=\sum_{i=1}^{h} h_{i}(x) x_{i} \cdot f .
$$

Applying $\alpha$ to (1) gives $\alpha(x \cdot f)=\sum_{i=1}^{n} h_{i}(x) \alpha\left(x_{i} \cdot f\right)$. Evaluating this at the identity element of $U(L)$ gives $\alpha(f)(x)=\sum_{i=1}^{n} h_{i}(x) \alpha\left(x_{i} \cdot f\right)(1)$ and so

$$
\alpha(f)=\sum_{i=1}^{n} \alpha\left(x_{i} \cdot f\right)(1) h_{i}
$$

Thus $\alpha(f)$ belongs to the space spanned by the right translates of $f$, which proves our first assertion.

Now suppose that $T$ and $\alpha$ are as in the second part of the lemma. We define a linear map $s: U \otimes U \rightarrow U \otimes U$ by $s\left(u_{1} \otimes u_{2}\right)=u_{1} u_{2}^{*}$, for all $u_{1}, u_{2} \in U(L)$. The composition $s \circ d$ gives the constant representative function 1 . This may be verified by evaluating $s \circ d$ at $u \in U(L)$, and arguing by induction on the filtration index of $u$.

From (1) it follows that, for arbitrary elements $x, y$, and $z$ in $U(L)$,

$$
\sum_{i=1}^{n} h_{i}\left(y^{*} x\right)\left(x_{i} \cdot f\right)(z)=f\left(z y^{*} x\right)
$$

and hence we see that

$$
\sum_{i=1}^{n}\left(\left(x \cdot h_{i}\right)^{*}\left(x_{i} \cdot f\right)\right)(u)=f((s \circ d)(u) x) .
$$

That is, that

$$
\sum_{i=1}^{n}\left(x \cdot h_{i}\right)^{*}\left(x_{i} \cdot f\right)
$$

is the constant representative function $f(x)$.

Suppose now that $\alpha(f)=0$. Then $\alpha\left(x_{i} \cdot f\right)=x_{i} \cdot \alpha(f)=0$ and so $f(x)=0$ for all $x \in U(L)$. Thus, $\alpha$ has kernel zero. $T$ is the sum of finite-dimensional subspaces which are stable under the right translations and so also stable under $\alpha$. Since the 
kernel of $\alpha$ is zero, $\alpha$ maps each of these subspaces onto itself and hence, $\alpha$ maps $T$ onto itself. This proves that $\alpha$ is an automorphism of $T$.

For $f \in T$, we define the function $\beta(f)$ on $U(L)$ by $\beta(f)(x)=\alpha\left((x \cdot f)^{*}\right)(1)$. Applying $\beta$ to (2), we obtain $\beta(\alpha(f))=\sum_{i=1}^{n} \alpha\left(x_{i} \cdot f\right)(1) \beta\left(h_{i}\right)$, which gives

$$
\beta(\alpha(f))(x)=\sum_{i=1}^{n} \alpha\left(x_{i} \cdot f\right)(1) \beta\left(h_{i}\right)(x)=\alpha\left(\sum_{i=1}^{n}\left(x \cdot h_{i}\right)^{*}\left(x_{i} \cdot f\right)(1)\right)=f(x) .
$$

That is, $\beta(\alpha(f))=f$ for each $f \in T$. Since $\alpha$ maps $T$ onto $T, \beta$ is an algebra endomorphism of $T$, and $\beta$ is the inverse of $\alpha$. This completes the proof of the lemma.

Let $\mathscr{H}$ be the group of all algebra automorphisms of $R(L)$ that commute with the left translations and leave $R_{s}$ fixed. Then $R(L)$ is a representation space for $\mathscr{H}$ in the natural way.

LEMMA 4.2. Every finite-dimensional subspace of $R(L)$ which is invariant under the right translations is a unipotent representation space for $\mathscr{H}$.

Proof. Note first that, by Lemma 4.1, each right $U(L)$-subspace of $R(L)$ is actually a representation space for $\mathscr{H}$. Let $S$ be a such a subspace of finite dimension and let $\{0\}=S_{0} \subset S_{1} \subset \cdots \subset S_{k}=S$ be a composition series for $S$ as a right $U(L)$-module. Choose $s_{i} \in S_{i}$ and let $t_{i}$ be a linear function on $S$ that vanishes on $S_{i-1}$. Then the function $t_{i} \mid s_{i}$, defined by $\left(t_{i} \mid s_{i}\right)(x)=t_{i}\left(s_{i} \cdot x\right)$ for each $x \in U(L)$, is a representative function associated with the anti-representation of $L$ by right translations on $S_{i} / S_{i-1}$, which is simple, and so $t_{i} \mid s_{i}$ belong to $R_{s}$. Hence $h\left(t_{i} \mid s_{i}\right)=t_{i} \mid s_{i}$ for each $h \in \mathscr{H}$.

If $e$ is a linear endomorphism of $R(L)$ commuting with the left translations then, again by Lemma 4.1, $e(S) \subset S$. We have $e(t \mid s)=t \mid s$ for each $s \in S$ and each linear function $t$ on $S$. It is clearly sufficient to prove this for the specific linear functions of the form $t_{u}$, which for each $u \in U(L)$, are defined by $t_{u}(s)=s(u)$ for all $s \in S$. In this case, $t_{u} \mid s=u \cdot s$, and for arbitrary $x \in U(L)$, we find:

$$
\begin{aligned}
e\left(t_{u} \mid s_{i}\right)(x) & =x \cdot e\left(t_{u} \mid s_{i}\right)(1) \\
& =e\left(x \cdot t_{u} \mid s_{i}\right)(1)=e\left(x u \cdot s_{i}\right)(1) \\
& =e\left(s_{i}\right)(x u)=\left(t_{u} \mid e\left(s_{i}\right)\right)(x) .
\end{aligned}
$$

By evaluating $t_{i}\left|h\left(s_{i}\right)=t_{i}\right| s_{i}$ at 1 , we conclude that $t_{i}\left(h\left(s_{i}\right)\right)=t_{i}\left(s_{i}\right)$ for each $h \in \mathscr{H}$. Since this holds for all $t_{i}$ that vanish on $S_{i-1}$, and since $S_{i}$ is $\mathscr{H}$-stable, this means that $h\left(s_{i}\right)-s_{i} \in S_{i-1}$ for all $h \in \mathscr{H}$. Thus, $\mathscr{H}$ is unipotently represented on S. q.e.d.

LEMMA 4.3. If $D$ is any normal basic subalgebra of $R(L)$, then $D^{N}=R(L)^{A} \otimes E$.

Proof. First we show that $R(L)^{N}=R(L)^{A} \otimes E \otimes C$. Clearly,

$$
R(L)^{A} \otimes E \otimes C \subset R(L)^{N} .
$$


Suppose then that $f \in R(L)^{N}$. Since by $[2$, p. 519]

$$
R(L)=R(L)^{A} \otimes C \otimes F\left[g_{1}, \cdots, g_{n}\right],
$$

we may write $f=\sum_{i=1}^{p} h_{i} \gamma_{i}$ with each $h_{i} \in R(L)^{A} \otimes C$, each $\gamma_{i} \in F\left[g_{1}, \cdots, g_{n}\right]$, and we may assume that the $h_{i}$ are linearly independent over $F . N \cdot f=\{0\}$ implies $N \cdot \gamma_{i}=\{0\}$ for each $i$, since $F\left[g_{1}, \cdots, g_{n}\right]$ is stable under the left translations. Hence, $\gamma_{i} \in E$ for each $i$, which proves the result. Since, for any field $F^{*} \supset F$, $N \otimes F^{*}$ is the nilpotent radical of $L \otimes F^{*}$ and $R\left(L \otimes F^{*}\right)^{N \otimes F^{*}}=R(L)^{N} \otimes F^{*}$, it suffices to prove the conclusion of the lemma in the case when $F$ is algebraically closed, which we shall now assume. $D$, being a left stable basic subalgebra, contains E. Hence,

$$
R(L)^{A} \otimes E \subset D^{N} \subset\left(R(L)^{A} \otimes E\right)[Q] .
$$

Since the elements of $Q$ are free over $D$, they are likewise free over $D^{N}$, so that this last result gives $D^{N}=R(L)^{A} \otimes E$. q.e.d.

Let $Z$ denote the center of $N$. Clearly, we may choose the basis $x_{1}, \cdots, x_{n}$ for $A$ so that in addition to the previous requirements, $x_{1}, \cdots, x_{p}$ forms a basis for $Z$. If $B$ again denotes the standard normal basic subalgebra, then

$$
B=R^{A}\left[g_{p+1}, \cdots, g_{n}\right]\left[g_{1}, \cdots, g_{p}\right] .
$$

Evidently, $R^{A} \subset B^{Z}$ and each $g_{k}$ with $k>p$ is in $B^{Z}$. Hence, $B=B^{Z}\left[g_{1}, \cdots, g_{p}\right]$. By "partial differentiation," it follows that the monomials in $g_{1}, \cdots, g_{p}$ are free over $B^{Z}$. Since

$$
R^{A}\left[g_{p+1}, \cdots, g_{n}\right] \subset B^{Z} \subset R^{A}\left[g_{p+1}, \cdots, g_{n}\right]\left[g_{1}, \cdots, g_{p}\right]
$$

this result implies that $B^{Z}=R^{A}\left[g_{p+1}, \cdots, g_{n}\right]$.

LEMMA 4.4. The free $B^{Z}$-module $B^{Z}+B^{Z} g_{1}+\cdots+B^{Z} g_{p}$ is stable under the left translations.

Proof. $Z$ is an ideal in $L$ and so $B^{Z}$ is stable under the left translations. Let $k$ be such that $1 \leqq k \leqq p$ and let $x \in L . F\left[g_{1}, \cdots, g_{n}\right]$ is stable under the left translations. In particular,

$$
x \cdot g_{k}=f\left(g_{1}, \cdots, g_{n}\right) \text { with } f \in F\left[g_{1}, \cdots, g_{n}\right] .
$$

To prove the lemma, it suffices to show that $f$ has the form $f=g_{0}+\sum_{i=1}^{p} u_{i} g_{i}$ with $u_{i} \in F\left[g_{p+1}, \cdots, g_{n}\right]$ for $i>0$ and $g_{0} \in F$. To show this, we need only show that when $u x_{n}^{e_{n}} \cdots x_{1}^{e_{1}} x$ is the expression in standard form (as a sum of ordered monomials), then each nonzero monomial $v x_{n}^{f_{n}} \ldots x_{1}^{f_{1}}$ appearing has the property that $f_{1}+\cdots+f_{p} \geqq e_{1}+\cdots+e_{p}$.

For $x \in H$, write

$$
u x_{n}^{e_{n}} \cdots x_{1}^{e_{1}} x=u x x_{n}^{e_{n}} \cdots x_{1}^{e_{1}}+u\left[x_{n}^{e_{n}} \cdots x_{1}^{e_{1}}, x\right] .
$$


For $x \in A$, write

$$
u x_{n}^{e_{n}} \cdots x_{1}^{e_{1}} x=u x_{n}^{e_{n}} \cdots x_{p+1}^{e_{p+1}} x x_{p}^{e_{p}} \cdots x_{1}^{e_{1}}+u x_{n}^{e_{n}} \cdots x_{p+1}^{e_{p+1}}\left[x_{p}^{e_{p}} \cdots x_{1}^{e_{1}}, x\right] .
$$

Since $Z$ is an abelian ideal in $L$, these formulas show that $u x_{n}^{e_{n}} \cdots x_{1}^{e_{1}}$ will have the required form, and the lemma is proved.

We shall say that an endomorphism of $R(L)$ is locally nilpotent (resp. locally unipotent) if its restriction to each finite-dimensional subspace of $R(L)$ is nilpotent (resp. unipotent). If $f$ is a locally nilpotent endomorphism of $R(L)$, then $\exp f=\sum_{n=0}^{\infty} f^{n} / n$ ! is a well-defined endomorphism of $R(L)$. If $f$ is a derivation of $R(L)$, then $\exp f$ is actually an algebra automorphism. If $f$ is a locally unipotent endomorphism of $R(L)$, then $\log f=\sum_{n=1}^{\infty}(-1)^{n+1}(1-f)^{n} / n$ is a well-defined endomorphism of $R(L)$. Each $x \in N$ acts, by right translation, as a locally nilpotent derivation of $R(L)$. Hence, the exponential of such a right translation is well defined and is an algebra automorphism of $R(L)$. We denote this automorphism by $E_{r}(x)$. Since $N$ is nilpotent, it follows from the Campbell-Hausdorff formula that, for $x$ and $y$ in $N$, the composition $E_{r}(x) \circ E_{r}(y)$ is equal to $E_{r}(f(x, y))$, where $f(x, y)-x-y$ is a sum of multiple commutators of $x$ and $y$. Hence, the functions $E_{r}(x)$ with $x \in N$ form a group, which we denote by $E_{r}(N)$. Each element of $E_{r}(N)$ leaves $R_{s}$ fixed, and sends basic subalgebras of $R(L)$ onto basic subalgebras.

Proposition 4.1. Let $\gamma$ be an algebra endomorphism of $R(L)$. Then $\gamma \in E_{r}(N)$ if and only if $\gamma$ commutes with the left translations and leaves the elements of $R^{N}$ fixed.

Proof. Clearly, the conditions of the proposition are necessary. Conversely, suppose that $\gamma$ is an algebra endomorphism of $R(L)$ commuting with left translations and leaving $R^{N}$ fixed. Then, by Lemma 4.1, $\gamma$ is an automorphism of $R(L)$, and by Lemma 4.2, $\gamma$ is locally unipotent. We assert that $\log \gamma$ is a derivation. To see this, let $f$ and $g$ be in $R(L)$ and choose an integer $m$ such that

$$
(\gamma-1)^{m+1}(f)=(\gamma-1)^{m+1}(g)=(\gamma-1)^{m+1}(f g)=0 .
$$

Let $t$ be an indeterminate and define $F(t) \in R(L)[t]$ by

$$
F(t)=\sum_{n=0}^{m}(n !)^{-1} t^{n}(\log \gamma)^{n}(f g)-\sum_{n=0}^{m}(n !)^{-1}(\log \gamma)^{n}(f) \cdot \sum_{n=0}^{m}(n !)^{-1} t^{n}(\log \gamma)^{n}(g) .
$$

Since $\gamma^{k}$ is an automorphism for every integer $k, F(k)=0$ for every integer $k$. Since $R(L)$ is an integral domain of characteristic zero, this means that $F(t)$ is the zero polynomial. Looking at the coefficient of the first degree term in $t$ shows that $\log \gamma$ is a derivation.

$\gamma$ commutes with the left translations, and therefore so does $\log \gamma \cdot \gamma$ leaves $R^{N}$ fixed, hence $\log \gamma$ annihilates $R^{N}$. Let $F^{*}$ denote the algebraic closure of $F$. We 
denote the canonical extension of $\log \gamma$ to a proper derivation of $R\left(L \otimes F^{*}\right)$ by $\overline{\log \gamma}$. Then $\overline{\log \gamma}$ annihilates $R(L)^{N} \otimes F^{*}=R\left(L \otimes F^{*}\right)^{N \otimes F^{*}}$ and, in particular, $(\overline{\log \gamma})(\exp f)=(\overline{\log \gamma})(f) \exp f=0$ for every elementary function $f$ on $U\left(L \otimes F^{*}\right)$. We conclude, using the right analog of [2, p. 519, Theorem 6] that $\log \gamma$ is the right translation by an element $x \in L . f \cdot x=0$ for each $f \in R^{A}$ and so $f(x)=0$ for $f \in R^{A}$. Hence $x \in A$, since the elements of $R^{A}$ separate the points of $H$. Further, we see that $x \in N$, since the elements of $F\left[g_{m+1}, \cdots, g_{n}\right]$ separate the points of $A / N$. Finally, the relation $E_{r}(\log \gamma)=\gamma$ completes the proof of the lemma.

We are now in a position to state and prove the conjugacy theorem for normal basic subalgebras.

THEOREM 4.1. Let $L$ be a Lie algebra over an arbitrary field of characteristic zero and let $V$ and $W$ be normal basic subalgebras of $R(L)$. Then there exists an element $\gamma \in E_{r}(N)$ such that $\gamma(W)=V$.

Proof. We assume first of all that the base field $F$ is algebraically closed, and proceed by induction on the dimension of $N$.

As before, let $Z$ be the center of $N$. If $Z=N$, we have $V^{Z}=W^{Z}$ by Lemma 4.3. If $Z \neq N$, we consider $L / Z$, identifying $R(L / Z)$ with $R(L)^{Z}$. Under this identification, the images of $V^{Z}$ and $W^{Z}$ are normal basic subalgebras of $R(L / Z)$. The nilpotent radical of $L / Z$ is $N / Z$.

In the case $N=\{0\}$, we have $V=W$ by Lemma 4.3 and so the result is true in this case. Hence, by assuming that the theorem is true in lower dimensions, we see that there is an element $x \in N$ such that $E_{r}(x)\left(W^{Z}\right)=V^{Z}$. Replacing $V$ by $E_{r}(-x)(V)$ we may assume that $W^{Z}=V^{Z}$, recalling that $E_{r}(N)$ is a group. For the same reason, it suffices to prove the theorem in the special case where $W=B$, the normal basic subalgebra introduced previously.

We may write each element $f \in R$ uniquely in the form $f=\Sigma_{q \in Q} v_{q}(f) q$ with each $v_{q}(f) \in V$. Then $v_{1}$ is a $U(L)$-module homomorphism of $R$ into $V$ and coincides with the identity on $B^{Z}=V^{Z}$. Denote by $\alpha$ the restriction of $v_{1}$ to $B^{Z}+B^{Z} g_{1}+\cdots+B^{Z} g_{p}$. Since $g_{1}, \cdots, g_{p}$ are algebraically free over $B^{Z}, \alpha$ extends uniquely to an algebra homomorphism $\beta: B \rightarrow V$. Further, we extend $\beta$ to an algebra endomorphism $\gamma$ of $R(L)$ such that $\gamma(q)=q$ for each $q \in Q$. Then it is clear from the definition of $\gamma$ and Lemma 4.4 that $\gamma$ commutes with the left translations and leaves the elements of $B^{N} \subset B^{Z}$ fixed. Hence, by Proposition 4.1, there is an element $x \in N$ such that $\gamma=E_{r}(x)$. This completes the proof in the case of an algebraically closed field.

Suppose now that $F$ is not necessarily algebraically closed, and let $F^{*}$ denote the algebraic closure of $F$. Since $F$ has characteristic zero, $F^{*}$ is a Galois extension of $F$. Let $G$ denote the Galois group of $F^{*}$ over $F$. $G$ acts as a group of $F$-algebra automorphisms of $U\left(L \otimes F^{*}\right)$ as follows : for $g \in G, l \in L$, and $a \in F^{*}$, $g(l \otimes a)=l \otimes g(a) . G$ then acts as a group of $F$-algebra automorphisms of 
$U\left(L \otimes F^{*}\right)^{\prime}$, where this action is given by $g(f)(u)=g\left(f\left(g^{-1}(u)\right)\right)$ for each $u \in U\left(L \otimes F^{*}\right), f \in U\left(L \otimes F^{*}\right)^{\prime}$, and $g \in G$. To show that such a map is multiplicative, first note that if, for $u \in U(L)$, we write $d(u)=\sum_{i=1}^{k}\left(a_{i} \otimes b_{i}\right)$ with each $a_{i}, b_{i} \in U(L)$, then $d\left(g^{-1}(u)\right)=\sum_{i=1}^{k}\left(g^{-1}\left(a_{i}\right) \otimes g^{-1}\left(b_{i}\right)\right)$ for each $g \in G$. Hence, for $f_{1}, f_{2} \in U\left(L \otimes F^{*}\right)^{\prime}$ we have

$$
\begin{aligned}
g\left(f_{1} f_{2}\right)(u) & =g\left\{\left(f_{1} f_{2}\right)\left(g^{-1}(u)\right)\right\} \\
& =g\left\{\left(f_{1} \otimes f_{2}\right)\left(\sum_{i=1}^{k} g^{-1}\left(a_{i}\right) \otimes g^{-1}\left(b_{i}\right)\right)\right\} \\
& =\sum_{i=1}^{k} g\left\{\left(f_{1}\left(g^{-1}\left(a_{i}\right)\right) f_{2}\left(g^{-1}\left(b_{i}\right)\right)\right\}\right. \\
& =\sum_{i=1}^{k} g\left(f_{1}\left(g^{-1}\left(a_{i}\right)\right)\right) g\left(f_{2}\left(g^{-1}\left(b_{i}\right)\right)\right) \\
& =\left(g\left(f_{1}\right) g\left(f_{2}\right)\right)(u) .
\end{aligned}
$$

By restriction, each $g \in G$ then induces an $F$-algebra automorphism of $R\left(L \otimes F^{*}\right)$, and by further restriction induces an automorphism of the multiplicative group $Q$.

Now given normal basic subalgebras $B$ and $V$ of $R(L)$, with $B$ as before, we may as above construct an automorphism $\gamma$ mapping $B \otimes F^{*}$ onto $V \otimes F^{*}$. We claim that $g \gamma=\gamma g$ for each $g \in G$. To show this, write $f \in R\left(L \otimes F^{*}\right)$ in the form $f=\Sigma_{q \in Q} v_{q}(f) q$ with each $v_{q}(f) \in V \otimes F^{*}$. Then $g(f)=\Sigma_{q \in Q} g\left(v_{q}(f)\right) g(q)$. Since $g(Q)=Q$ and $g(1)=1$, we conclude from the uniqueness of the expansion of $g(f)$ as a $V \otimes F^{*}$-linear combination of the elements of $Q$ that $\left(v_{1} g\right)(f)=\left(g v_{1}\right)(f)$. Hence, $(\gamma g)(f)=(g \gamma)(f)$ for each $f \in B \otimes F^{*}$. It follows that $\gamma(B) \subset\left(V \otimes F^{*}\right)^{G}=V$ and so $\gamma(B)=V$ since $\gamma(B)$ is also a normal basic subalgebra of $R(L)$.

As before, $\log \gamma$ is the right translation by an element $x \in N \otimes F^{*}$. By the definition of the action of $G$, we have $g(f \cdot x)=g(f) \cdot g(x)$ for every $f \in R\left(L \otimes F^{*}\right)$ and every $g \in G$. Since $\gamma$ commutes with the $G$-action, as does $\log \gamma$, whence $g(f \cdot x)=g(f) \cdot x$ for every $f \in R\left(L \otimes F^{*}\right)$ and every $g \in G$. Hence,

$$
g(f) \cdot x=g(f) \cdot g(x),
$$

and so the right translation on $R\left(L \otimes F^{*}\right)$ by $x-g(x)$ is 0 , whence $x=g(x)$ for every $g \in G$. That is, $x\left(\in N \otimes F^{*}\right)^{G}=N$. This completes the proof of the theorem.

5. The proper automorphisms of $R(L)$. We begin by recalling somedefinitions from [2]. Let $M$ be a finite-dimensional vector space over $F$, and $P_{M}$ the algebra of all polynomial functions on $E_{M}$. For a representation $\rho$ of a Lie algebra $L, R(\rho)$ denotes the space of the representative functions associated with $\rho$ and $S_{\rho}$ denotes the subalgebra of $R(L)$ generated by the constants and the elements of $R(\rho)$. 
If $\rho$ is a representation of $L$ in $E_{M}$, the map $t \rightarrow t \circ \rho$ of $E_{M}{ }^{\prime}$ onto $R(\rho)$ extends uniquely to a unitary algebra epimorphism of $P_{M}$ onto $S_{\rho}$, which is denoted by $p \rightarrow \hat{p}$. The kernel of this epimorphism is called the ideal associated with $\rho$, and is denoted by $Q_{M}$.

Proposition 5.1. Let $S$ be a finite-dimensional subspace of $R(L)$ that is stable under the right and left translations. Let $\hat{S}$ be the subalgebra of $R(L)$ that is generated by the constants and the elements of $S$. Let $\mathscr{A}$ be the group of all proper automorphisms of $\hat{S}$ and let $\mathscr{A}_{s}$ be its restriction image in the group of all linear automorphisms of $S$. Let $Q_{s}$ be the ideal associated with the representation $\rho_{s}$ of $U(L)$ by left translations on $S$. Let $G_{s}$ be the algebraic subgroup of $E_{s}$ determined by $Q_{s}$. Then $\mathscr{A}_{s}=G_{s}$.

Proof. It is easily seen that $\hat{S}=S_{\rho}$. We note that $e\left(t \circ \rho_{s}\right)=(e \cdot t) \circ \rho_{s}$ for every $t \in E_{s}^{\prime}$ and every $e \in E_{s}$ commuting with the right translations. To check this, define for each $x \in U(L)$ and each $s \in S$, the element $x^{\prime} / s \in E_{s}^{\prime}$ by $\left(x^{\prime} / s\right)(e)=e(s)(x)$. Since each $t \in E_{s}^{\prime}$ may be written as an $F$-linear combination of the $x^{\prime} / s$, it is sufficient to verify the above identity for a special $t$ of the form $x^{\prime} / s$. In this case the result is immediate.

Now we show that $\mathscr{A}_{s} \subset G_{s}$. For $\alpha \in \mathscr{A}$, denote by $\alpha_{s}$ the restriction of $\alpha$ to $S$. Then it suffices to show that $\alpha_{s} \cdot Q_{s} \subset Q_{s}$ for each $\alpha \in \mathscr{A}$. Suppose therefore that $p\left(t_{1}, \cdots, t_{n}\right) \in Q_{s}$, with each $t_{i} \in E_{s}^{\prime}$, and that $\alpha \in \mathscr{A}$. Then $p\left(t_{1} \circ \rho_{s}, \cdots, t_{n} \circ \rho_{s}\right)=0$ as an element of $\hat{S}$. Hence, since $\alpha_{s} \cdot p\left(t_{1}, \cdots, t_{n}\right)=p\left(\alpha_{s} \cdot t_{1}, \cdots, \alpha_{s} \cdot t_{n}\right)$, we see that the image of $\alpha_{s} \cdot p\left(t_{1}, \cdots, t_{n}\right)$ under $p \rightarrow \hat{p}$ is

$$
\begin{aligned}
p\left(\left(\alpha_{s} \cdot t_{1}\right) \circ \rho_{s}, \cdots,\left(\alpha_{s} \cdot t_{n}\right) \circ \rho_{s}\right) & =p\left(\alpha_{s}\left(t_{1} \circ \rho_{s}\right), \cdots, \alpha_{s}\left(t_{n} \circ \rho_{s}\right)\right) \\
& =\alpha\left(p\left(t_{1} \circ \rho_{s}, \cdots, t_{n} \circ \rho_{s}\right)\right)=0 .
\end{aligned}
$$

This shows that $\alpha s \cdot p\left(t_{1}, \cdots, t_{n}\right) \in Q_{s}$, as required.

Next we show that $G_{s} \subset \mathscr{A}_{s}$. Let $e \in G_{s}$. If $x$ and $y$ are fixed elements of $U(L)$ and $s \in S$, then the linear function $u \rightarrow u(s \cdot y)(x)-u(s)(y x)$ on $E_{s}$ is in $Q_{s}$, hence vanishes at $e$. This shows that $e$ commutes with the right translations. Since the the map $p \rightarrow \hat{p}$ is an epimorphism of $P_{s}$ onto $\hat{S}$, every element of $\hat{S}$ may be in the form $p\left(t_{1} \circ \rho_{s}, \cdots, t_{n} \circ \rho_{s}\right)$ with each $t_{i} \in E_{s}^{\prime}$. Suppose that $p\left(t_{1} \circ \rho_{s}, \cdots, t_{n} \circ \rho_{s}\right)$ is the zero element of $\hat{S}$. Then $p\left(t_{1}, \cdots, t_{n}\right) \in Q_{s}$. If $e \in G_{s}$, then $e \cdot Q_{s}=Q_{s}$, and in particular $e \cdot p\left(t_{1}, \cdots, t_{n}\right)=p\left(e \cdot t_{1}, \cdots, e \cdot t_{n}\right) \in Q_{s}$. Hence,

$$
p\left(e\left(t_{1} \circ \rho_{s}\right), \cdots, e\left(t_{n} \circ \rho_{s}\right)\right)=p\left(\left(e \cdot t_{1}\right) \circ \rho_{s}, \cdots,\left(e \cdot t_{n}\right) \circ \rho_{s}\right)=0 .
$$

This shows that $e$ may be extended (uniquely) to an algebra endomorphism $e^{+}$of $\hat{S}$ that leaves the constants fixed. Likewise, we can find an extension $\left(e^{-1}\right)^{+}$of $e^{-1}$ to an algebra endomorphism of $S$ leaving the constants fixed. The compositions $e^{+}\left(e^{-1}\right)^{+}$and $\left(e^{-1}\right)^{+} e^{+}$are algebra endomorphisms of $\hat{S}$ which coincide 
with the identity on the constants and on the elements of $S$, hence, are both equal to the identity automorphism of $\hat{S}$. Also, $e^{+}$commutes with the right translations since $e$ does. Thus $e^{+}$is a proper automorphism of $\hat{S}$. Since $\left(e^{+}\right)_{s}=e$, we conclude that $e \in \mathscr{A}_{s}$. This completes the proof of the proposition.

Now assume that $F$ is algebraically closed. Let $\mathscr{S}$ be the family of all finitedimensional, two-sidedly stable subspaces of $R(L)$. For each $S \in \mathscr{S}$ we construct $\mathscr{A}_{s}$ as in Proposition 5.1. Since each $Q_{s}$ is a prime ideal, each $\mathscr{A}_{s}$ is an irreducible algebraic subgroup of the corresponding general linear group.

Let $U$ and $V$ be in $\mathscr{S}$, with $U \supset V$. The restriction map $h_{v}^{u}: \mathscr{A}_{u} \rightarrow \mathscr{A}_{v}$ is a rational group homomorphism, and so by $[1$, p. 122 , Corollary 1$]$ it follows that $h_{v}^{u}\left(\mathscr{A}_{u}\right)$ is an algebraic subgroup of $\mathscr{A}_{v}$. Using [2, p. 503, Theorem 1], and [2, pp. 511-512], we see that the differential of $h_{v}^{u}$ maps the Lie algebra of $\mathscr{A}_{u}$ onto the Lie algebra of $\mathscr{A}_{v}$. Hence, since $\mathscr{A}_{v}$ is irreducible, $h_{v}^{u}$ maps $\mathscr{A}_{u}$ onto $\mathscr{A}_{v}$.

Thus we have an inverse system $\left(\mathscr{A}_{u}, h_{v}^{u}\right)$ of linear algebraic groups and rational group epimorphisms $h_{v}^{u}: \mathscr{A}_{u} \rightarrow \mathscr{A}_{v}$ satisfying the conditions $h_{w}^{v} \circ h_{v}^{u}=h_{w}^{u}$ for $U \supset V \supset W$. This inverse system defines an inverse limit group $\mathscr{A}$. Since every finite subset of $R(L)$ belongs to some $S \in \mathscr{S}$, it is clear that $\mathscr{A}$ may be identified with the group of all proper automorphisms of $R(L)$.

Noting the result of $[4$, p. 505, Proposition 2.8] we arrive at

THEOREM 5.1. Let L be a Lie algebra over an algebraically closed field of characteristic zero, and let $R(L)$ be the algebra of the representative functions on L. Let $T$ be a finitely generated subalgebra of $R(L)$ that contains the constants and is stable under the right and left translations. Then every proper automorphism of $T$ is the restriction to $T$ of a proper automorphism of $R(L)$.

Let $S, \hat{S}, G_{s}$ and $p \rightarrow \hat{p}$ be as before. Let $P\left(G_{s}\right)$ denote the algebra of polynomial functions on $G_{s}$. Denote by $p \rightarrow \bar{p}$ the canonical epimorphism $P_{s} \rightarrow P\left(G_{s}\right)$. The epimorphism $p \rightarrow \hat{p}$ induces an isomorphism $\bar{p} \rightarrow(\bar{p})^{\wedge}=\hat{p}$ of $P\left(G_{s}\right)$ onto $\hat{S}$. We define a family of functions $(\hat{S})^{\#}$ on $G_{s}$ by $f^{\#}(\alpha)=\alpha(f)(1)$ for each $f \in \hat{S}$ Then $f \rightarrow f^{\#}$ is a unitary algebra epimorphism of $\hat{S}$ onto $(\hat{S})^{\#}$. For any group $G$ and any function $f$ on $G$, we define a function $f^{4}$ on $G$ by $f^{\zeta}(g)=f\left(g^{-1}\right)$ for each $g \in G$.

Proposition 5.2. Let $S, \hat{S}$, and $G_{s}$ be as above. Then the set of functions $(\hat{S})^{\#}$ is precisely the algebra of all polynomial functions on $G_{s}$. The algebra epimorphism $\hat{S} \rightarrow(\hat{S})^{\#}$ defined above is a monomorphism, and is in fact the inverse of the previously defined algebra isomorphism $P\left(G_{s}\right) \rightarrow S$. If, in addition, $S$ is stable under the involution $f \rightarrow f^{*}$, then the inverse of the determinant function on $G_{s}$ lies in $P\left(G_{s}\right)$, and so every rational representation of $G_{s}$ is a polynomial representation.

Proof. We note that for each $x \in U(L)$ and $s \in S, \overline{x^{\prime} / s}=(s \cdot x)^{\#}$. The constants and the elements $\overline{x^{\prime} / s}$ for $x \in U(L)$ and $s \in S$ form a set of algebra generators for 
$P\left(G_{s}\right)$, while the constants and the elements $s^{\#}$ with $s \in S$ form a set of algebra generators for $(\hat{S})^{\#}$. Thus $P\left(G_{s}\right)=(\hat{S})^{\#}$. Let $\gamma \in G_{s}$. Then

$$
\left(\overline{\left(x^{\prime} / s\right)^{\wedge}}\right)^{\#}(\gamma)=\gamma\left(\left(\overline{\left.x^{\prime} / s\right)^{\wedge}}\right)(1)=\gamma\left(x^{\prime} / s \circ \rho_{s}\right)(1)=\gamma(s \cdot x)(1)=\gamma(s)(x)=\overline{\left(x^{\prime} / s\right)}(\gamma)\right.
$$

It follows that $(\hat{p})^{\#}=p$ for each $p \in P\left(G_{s}\right)$. Now let $t \in S$. Then $t^{\#}=\overline{1^{\prime} / t}$, whence, for every $x \in U(L)$,

$$
t^{\# \wedge}(x)=\left(1^{\prime} / t\right)\left(\rho_{s}(x)\right)=(x \cdot t)(1)=t(x) .
$$

Thus $t^{\# \wedge}=t$. Again, this extends to each $t \in \hat{S}$. We have shown that $p \rightarrow p^{\#}$ and $p \rightarrow \hat{p}$ are mutually inverse isomorphisms.

Now suppose that $S$ is stable under the involution $f \rightarrow f^{*}$. Using the last statement of the right analog of Lemma 4.1, we see that $f^{\# \zeta}=f^{* *}$ for each $f \in S$ and so, in particular, that $(\hat{S})^{\#}$ is stable under the involution $f \rightarrow f^{4}$. But if $d$ is the determinant function on $G_{s}$, then $d^{-1}=d^{\natural}$. The last statement of the proposition follows from the fact that the representative functions associated with any rational representation of $G_{s}$ lie in $P\left(G_{s}\right)\left[d^{-1}\right]$. This completes the proof.

Let $\mathscr{A}$ again denote the group of the proper automorphisms of $R(L)$. We define, for each $f \in R(L)$, the function $f^{\#}$ on $\mathscr{A}$ by $f^{\#}(\alpha)=\alpha(f)(1)$ for each $\alpha \in \mathscr{A}$. The algebra of functions obtained from $R(L)$ by the algebra homomorphism $f \rightarrow f^{\#}$ is denoted by $R(L)^{\#}$. We have the following results:

THEOREM 5.2. The map $R(L) \rightarrow R(L)^{\#}$ is an algebra isomorphism. The algebra $R(L){ }^{*}$ of functions on $\mathscr{A}$ is two-sidedly stable under translations, and is stable under the mapping $f \rightarrow f^{4}$.

Proof. The first statement follows from Theorem 5.1 and Proposition 5.2. Stability under $f \rightarrow f^{4}$ follows as in Proposition 5.2, stability under left translations is immediate, and stability under right translations follows from this and the relation $f \cdot \alpha=\left(\alpha^{-1} \cdot f^{\zeta}\right)^{\zeta}$ which holds for each $\alpha \in \mathscr{A}$ and each $f \in R(L)$.

In the previous section, $E_{r}(N)$ was defined by considering exponentials of right translations. Analogously, we may consider exponentials of left translations and so obtain a group $E_{l}(N)$ of proper automorphisms of $R(L)$.

THEOREM 5.3. $E_{l}(N)$ is normal subgroup of $\mathscr{A} . \mathscr{A}$ admits a semidirect product decomposition $\mathscr{A}=\mathscr{A}_{L / A} . \mathscr{B}$ where $\mathscr{A}_{L / A}$ is isomorphic, via restriction, with the group of all proper automorphisms of $R(L / A)$ and $\mathscr{B}$ is a semidirect product $\mathscr{A}_{L /[L, L]} \cdot E_{l}(N)$, where $\mathscr{A}_{L /[L, L]}$ is isomorphic, via restriction, with the group of all proper automorphisms of $R(L /[L, L])$. Furthermore, $\mathscr{A}_{L /[L, L]}$ is isomorphic with the direct product of an $(n-m)$-dimensional vector group and the group of the proper automorphisms of $C$. 
Proof. It was shown in $[2$, p. 519] that

$$
R(L)=R(L)^{A} \otimes C \otimes E \otimes F\left[g_{1}, \cdots, g_{m}\right] .
$$

Since $R(L)^{A}, C$, and $E$ are stable under the involution $f \rightarrow f^{*}$ it follows that

$$
R(L)=R(L)^{A} \otimes C \otimes E \otimes F\left[g_{1}, \cdots, g_{m}\right]^{*} .
$$

$R(L)^{A}$ is isomorphic with $R(L / A)$ and is stable under the proper automorpisms of $R(L)$. Let $\mathscr{A}_{L / A}$ be the subgroup of $\mathscr{A}$ consisting of those automorphisms which coincide with the identity on $C \otimes E \otimes F\left[g_{1}, \cdots, g_{m}\right]^{*}$. Since $C \otimes E \otimes F\left[g_{1}, \cdots, g_{m}\right]^{*}$ is stable under the right translations, every proper automorphism of $R(L)^{A}$ can be extended to an element of $\mathscr{A}_{L / A}$. It follows that $\mathscr{A}_{L / A}$ is isomorphic with the group of the proper automorphisms of $R(L / A)$ and that $\mathscr{A}$ is a semidirect product $\mathscr{A}_{L / A} \cdot \mathscr{B}$ where $\mathscr{B}$ is the subgroup of $\mathscr{A}$ consisting of those automorphisms which coincide with the identity on $R(L)^{A}$.

The subalgebra $E$ is two-sidedly stable and hence stable under the proper automorphisms of $R(L)$. Moreover, if $g$ is any elementary function and $\alpha$ is a proper automorphism of $E$, then $\alpha(g)=g+c$ with $c \in F$. In order to see this, note first that $f \in R(L)$ is elementary if and only if $f$ vanishes on $F$ and $L^{2} U(L)$. Let $x, y \in L$ and $u \in U(L)$. Then $\alpha(g)(x y u)=\alpha(g \cdot x y)(u)=\alpha(0)(u)=0$, whence $\alpha(g)$ vanishes on $L^{2} U(L)$. This gives $\alpha(g)=g^{\prime}+c$ where $g^{\prime} \in E$ and $c \in F$. For each $x \in L, g \cdot x$ is a constant function, hence $\alpha(g)(x)=\alpha(g \cdot x)(1)=g(x)$ and so $g^{\prime}=g$, which proves the assertion. Since, given $c_{m+1}, \cdots, c_{n} \in F$, the map $g_{i} \rightarrow g_{i}+c_{i}, i=m+1, \cdots, n$, can evidently be extended to a proper automorphism of $E=F\left[g_{m+1}, \cdots, g_{n}\right]$, we have shown that the group of the proper automorphisms of $E$ is isomorphic with $F^{n-m}$.

By [2, p. 519], $R(L /[L, L]) \approx C \otimes E . C \otimes E$ is stable under the proper automorphisms of $R(L)$. Let $\mathscr{A}_{L /[L, L]}$ be the subgroup of $\mathscr{B}$ consisting of those automorphisms which coincide with the identity on $F\left[g_{1}, \cdots, g_{m}\right]^{*}$. Now suppose, for the moment, that $F$ is algebraically closed, and let $\alpha$ be a proper automorphism of $C \otimes E$. Since $E$ is finitely generated, we know from Theorem 5.1 that there is an element $\beta$ in $\mathscr{A}$ such that $\beta$ coincides with $\alpha$ on $E$. Since $R(L)^{A} \otimes E \otimes F\left[g_{1}, \cdots, g_{m}\right]^{*}$ is right stable we can find $\gamma \in \mathscr{A}$ such that $\gamma$ coincides with $\alpha \beta^{-1}$ on $C$ and with the identity on $R(L)^{A} \otimes E \otimes F\left[g_{1}, \cdots, g_{m}\right]^{*}$. Then $\gamma \beta \in \mathscr{A}$ and is an extension of $\alpha$. Since $\mathscr{A}=\mathscr{A}_{L / A} \cdot \mathscr{B}$, this shows that each proper automorphism of $C \otimes E$ may be extended to an element of $\mathscr{B}$. It follows that $\mathscr{A}_{L /[L, L]}$ is isomorphic with the group of the proper automorphisms of $R(L /[L, L])$ and that $\mathscr{B}$ is a semidirect product $\mathscr{A}_{L /[L, L]} \cdot X$, where $X$ is the subgroup of $\mathscr{A}$ consisting of those proper automorphisms of $R(L)$ which coincide with the identity on $R(L)^{A} \otimes C \otimes E$. But we showed in proving Lemma 4.3 that $R(L)^{A} \otimes C \otimes E=R(L)^{N}$. Hence, by the right analog of Proposition 4.1, it follows that, whenever $F$ is algebraically closed, $\mathscr{B}=\mathscr{A}_{L /[L, L]} \cdot E_{l}(N)$. 
Now let $F$ be a field that is not necessarily algebraically closed and let $\bar{F}$ be it algebraic closure. Let $\bar{L}=L \otimes \bar{F}$ and $\bar{N}=N \otimes \bar{F}$, and as usual consider $R(L)$ as a subalgebra of $R(\bar{L})$. Denote by $\overline{\mathscr{A}}$ the group of the proper automorpisms of $R(\bar{L})$. Each $\alpha \in \mathscr{A}$ extends uniquely to a proper automorphism $\bar{\alpha}$ of $R(\bar{L})$ leaving $R(L)$ stable. Let $\beta \in \mathscr{B}$. Then, by the above, $\bar{\beta}=\bar{\gamma} \circ \bar{\delta}$, with $\bar{\gamma} \in \overline{\mathscr{A}}_{\bar{L} /[\bar{L}, \bar{L}]}$ and $\bar{\delta} \in E_{l}(\bar{N})$. Clearly $\bar{\gamma}$ and $\bar{\delta}$ leave $R(L)$ stable, so by restriction induce elements $\gamma$ and $\delta$ of $\mathscr{A}$. Furthermore, $\gamma \in \mathscr{A}_{L /[L, L]}$ and $\delta \in E_{l}(N)$. Hence, in general, we have $\mathscr{B}=\mathscr{A}_{L /[L, L]} \cdot E_{l}(N)$.

Also, $E_{l}(N)$ is normal in $\mathscr{A}$ since it is the kernel of the restriction homomorphism from $\mathscr{A}$ to the group of the proper automorphisms of $R(L)^{N}$. Using the twosided stability of both $C$ and $E$, the result about the structure of $\mathscr{A}_{L /[L, L]}$ follows from what we have shown above.

REMARK. Since $L / A$ is semisimple, $\mathscr{A}_{L / A}$ is isomorphic with an irreducible algebraic linear group whose Lie algebra may be identified with $L / A$. Indeed this is clear from Proposition 5.1 and $[2, \S 5]$.

6. On inverse limits of algebraic groups. In this section, $F$ is assumed to be algebraically closed. Let $(\mathscr{D},<)$ be a directed set, and suppose that we are given an inverse system $h_{\alpha}^{\beta}: G_{\beta} \rightarrow G_{\alpha}$ of linear algebraic groups $G_{\alpha}$ and rational group epimorphisms $h_{\alpha}^{\beta}$ defined for every pair of elements $\alpha$ and $\beta$ such that $\alpha<\beta$. Let $\mathscr{G}$ denote the inverse limit group defined by this system. By [4, p. 505, Proposition 2.8], the natural homomorphism $h_{\alpha}: \mathscr{G} \rightarrow G_{\alpha}$ is an epimorphism for each $\alpha \in \mathscr{D}$. We have $h_{\alpha}=h_{\alpha}^{\beta} \circ h_{\beta}$ for each pair $\alpha, \beta \in \mathscr{D}$ with $\alpha<\beta$.

Let $R\left(G_{\alpha}\right)$ denote the algebra of the rational representative functions on $G_{\alpha}$. We define algebra monomorphisms $\pi_{\beta}^{\alpha}: R\left(G_{\alpha}\right) \rightarrow R\left(G_{\beta}\right)$ for each pair $\alpha<\beta$ by $\pi_{\beta}^{\alpha}(f)=f \circ h_{\alpha}^{\beta}$ for each $f \in R\left(G_{\alpha}\right)$. This is a direct system of algebras $R\left(G_{\alpha}\right)$ and algebra monomorphisms $\pi_{\beta}^{\alpha}$. When the $R\left(G_{\alpha}\right)$ are considered only on $F$-spaces, this system yields a direct limit $F$-space $R(\mathscr{G})$ and natural $F$-linear maps $\pi_{\alpha}: R\left(G_{\alpha}\right) \rightarrow R(\mathscr{G})$ for each $\alpha \in \mathscr{D}$. The $\pi_{\alpha}$ are known to be monomorphisms since the $\pi_{\beta}^{\alpha}$ are monomorphisms. Also, $\pi_{\beta} \circ \pi_{\beta}^{\alpha}=\pi_{\alpha}$ for each pair $\alpha<\beta$. Since $\mathscr{D}$ is directed, $R(\mathscr{G})=\bigcup_{\alpha \in \mathscr{D}} \pi_{\alpha}\left(R\left(G_{\alpha}\right)\right)$.

We will now define an algebra structure on $R(\mathscr{G})$. For arbitrary elements $f$, $g \in R(\mathscr{G})$, choose $\alpha \in \mathscr{D}$ and $f^{\alpha} \in R\left(G_{\alpha}\right)$ such that $f=\pi_{\alpha}\left(f^{\alpha}\right)$, and choose $\beta \in \mathscr{D}$ and $g^{\beta} \in R\left(G_{\beta}\right)$ such that $g=\pi_{\beta}\left(g^{\beta}\right)$. Choose $\gamma \in \mathscr{D}$ such that $\alpha<\gamma$ and $\beta<\gamma$. Define the product of $f$ and $g$ by

$$
f g=\pi_{\gamma}\left(\pi_{\gamma}^{\alpha}\left(f^{\alpha}\right) \cdot \pi_{\gamma}^{\beta}\left(g^{\beta}\right)\right) .
$$

It is easy to check that this product is independent of the choices of $\alpha, \beta, f^{\alpha}$, and $g^{\beta}$ and hence, that it is associative and distributive. Clearly, each $\pi_{\alpha}$ is multiplicative with respect to this product. Let $\mathscr{F}(\mathscr{G})$ denote the algebra of all $F$ valued functions on $\mathscr{G}$.

Define a unitary algebra homomorphism $i: R(\mathscr{G}) \rightarrow \mathscr{F}(\mathscr{G})$ by $i(f)=f^{\alpha} \circ h_{\alpha}$ 
where $\alpha$ and $f^{\alpha}$ are chosen so that $f=\pi_{\alpha}\left(f^{\alpha}\right)$. Clearly it is well defined and, since $\mathscr{D}$ is directed and each $\pi_{\alpha}$ is a monomorphism, $i$ is a monomorphism. By means of $i$, we identify $R(\mathscr{G})$ with a subalgebra of $\mathscr{F}(\mathscr{G})$.

DEFINITION. $R(\mathscr{G})$ is called the algebra of the rational representative functions on $\mathscr{G}$.

REMARK. In the notation of the previous section, it is clear that $R(L){ }^{\#}$ is precisely the algebra of the rational representative functions on $\mathscr{A}$.

For each $\alpha \in \mathscr{D}$, let $L_{\alpha}$ denote the Lie algebra of $G_{\alpha}$, and for each $\alpha>\beta$, let $d h_{\beta}^{\alpha}$ denote the differential of $h_{\beta}^{\alpha}$. Then, since $F$ has characteristic zero, $d h_{\beta}^{\alpha}$ is a Lie algebra epimorphism of $L_{\alpha}$ onto $L_{\beta}$. The inverse system $\left(L_{\alpha}, d h_{\beta}^{\alpha}\right)$ defines an inverse limit Lie algebra which we denote by $\mathscr{L}$. By $[4$, p. 505, Proposition $2.10]$, the Lie algebra homomorphisms $d h_{\alpha}: \mathscr{L} \rightarrow L_{\alpha}$ are epimorphisms.

We recall that if $H$ is an algebraic group of automorphisms of a vector space $V$ and if $e$ belongs to the Lie algebra of $H$, then $D_{e}$ denotes the proper derivation of $R(H)$ that is induced by the left translation by $e$ on $E_{v}^{\prime}$.

Let $E_{R}$ denote the algebra of endomorphisms of $R(\mathscr{G})$ and define a Lie algebra monomorphism $i: \mathscr{L} \rightarrow E_{R}$ as follows: given $l \in \mathscr{L}$ and $f \in R(\mathscr{G})$, choose $\alpha \in \mathscr{D}$ and $f^{\alpha} \in R\left(G_{\alpha}\right)$ such that $\pi_{\alpha}\left(f^{\alpha}\right)=f$, and define $i(l)$ by $i(l)(f)=\pi_{\alpha}\left(D_{d h_{\alpha}(l)}\left(f^{\alpha}\right)\right)$. We will show that $i(l)(f)$ is independent of $\alpha$. For suppose that we have $\alpha, \beta \in \mathscr{D}$, $f^{\alpha} \in R\left(G_{\alpha}\right)$, and $f^{\beta} \in R\left(G_{\beta}\right)$ such that $\pi_{\alpha}\left(f^{\alpha}\right)=\pi_{\beta}\left(f^{\beta}\right)=f$. We must show that $\pi_{\alpha}\left(D_{d h_{\gamma}(l)}\left(f^{\alpha}\right)\right)=\pi_{\beta}\left(D_{d h_{\beta}(l)}\left(f^{\beta}\right)\right)$. It clearly suffices to do this in the case $\alpha<\beta$. Then:

$$
\begin{aligned}
\pi_{\alpha}\left(D_{d h_{\alpha}(l)}\left(f^{\alpha}\right)\right)-\pi_{\beta}\left(D_{d h_{\beta}(l)}\left(f^{\beta}\right)\right) & =\pi_{\beta}\left(\pi_{\beta}^{\alpha}\left(D_{d h_{\alpha}(l)}\left(f^{\alpha}\right)-D_{d h_{\beta}(l)}\left(f^{\alpha}\right)\right)\right. \\
& =\pi_{\beta}\left(D_{d h_{\alpha}(l)}\left(f^{\alpha}\right) \circ h_{\alpha}^{\beta}-D_{d h_{\beta}(l)}\left(f^{\beta}\right)\right) \\
& =\pi_{\beta}\left(D_{d h_{\alpha}^{\beta}\left(d h_{\beta}(l)\right)}\left(f^{\alpha}\right) \circ h_{\alpha}^{\beta}-D_{d h_{\beta}(l)}\left(f^{\alpha} \circ h_{\alpha}^{\beta}\right)\right) .
\end{aligned}
$$

But $D_{\left.d h_{\alpha}^{\beta} d h_{\beta}(l)\right)}\left(f^{\alpha}\right) \circ h_{\alpha}^{\beta}=D_{d h_{\beta}(l)}\left(f^{\alpha} \circ h_{\alpha}^{\beta}\right)$ by the definition of the differential of a rational map and so the required result follows.

It is easily checked that the commutativity relations $\pi_{\alpha}\left(h_{\alpha}(g) \cdot f^{\alpha}\right)=g \cdot \pi_{\alpha}\left(f^{\alpha}\right)$ and $\pi_{\alpha}\left(f^{\alpha} \cdot h_{\alpha}(g)\right)=\pi_{\alpha}\left(f^{\alpha}\right) \cdot g$ are valid for each $f^{\alpha} \in R\left(G_{\alpha}\right)$ and each $g \in \mathscr{G}$.

We now show that $i(\mathscr{L})$ is precisely the algebra of the proper derivations of $R(\mathscr{G})$. First, each element of $i(\mathscr{L})$ is clearly a derivation of $R(\mathscr{G})$ which, by the commutativity relations, commutes with the right translations. Let $D$ be any proper derivation of $R(\mathscr{G})$. The commutativity relations show that, for any $f \in R(\mathscr{G})$, $\mathscr{G} \cdot f$ (and $f \cdot \mathscr{G}$ ) have finite dimension. Hence, an argument as used in proving the first part of Lemma 4.1 shows that each subspace of $R(\mathscr{G})$ that is stable under the left translations is also stable under $D$. Again by the commutativity relations, each $\pi_{\alpha}\left(R\left(G_{\alpha}\right)\right)$ is stable under the left translations. Hence $D$ induces a proper derivation of each $\pi_{\alpha}\left(R\left(G_{\alpha}\right)\right)$ and so of $R\left(G_{\alpha}\right)$. This derivation has the form $D_{l_{\alpha}}$ for some (unique) $l_{\alpha} \in L_{\alpha}$. It follows directly that for $\alpha<\beta$, we have $d h_{\alpha}^{\beta}\left(l_{\beta}\right)=l_{\alpha}$. Hence the set $\left\{l_{\alpha}\right\}_{\alpha \in \mathscr{D}}$ determines an element $l \in \mathscr{L}$. Clearly, $i(l)=D$ and we 
have proved that $i(\mathscr{L})$ coincides with the Lie algebra of the proper derivations of $R(\mathscr{G})$. By means of $i$, we identify $\mathscr{L}$ with this algebra.

Definitions. Let $(\rho, V)$ be a representation of $\mathscr{G}$, not necessarily finite-dimensional. For each $\mathscr{G}$-stable finite-dimensional subspace $S$ of $V$, let $\rho_{s}$ denote the induced representation on $S$. Then the algebra generated by the functions $\lambda \circ \rho_{s}$, where $S$ ranges over the finite-dimensional $\mathscr{G}$-invariant subspaces of $V$ and $\lambda$ ranges over $E_{s}^{\prime}$ for each $S$, is called the algebra of representative functions associated with $\rho$, and denoted by $R(\rho)$. If $R(\rho) \subset R(\mathscr{G}), \rho$ is said to be a rational representation of $\mathscr{G} . \rho$ is said to be a locally finite representation if, for each $v \in V$, $\rho(\mathscr{G})(v)$ is contained in a finite-dimensional subspace of $V$. A locally finite representation of $\mathscr{L}$ is a similarly defined.

We shall now associate with every locally finite representation $(\rho, V)$ of $\mathscr{G}$ a locally finite representation $(d \rho, V)$ of $\mathscr{L}$. Assume first that $V$ is of finite dimension. In that case, $R(\rho) \subset R(\mathscr{G})$ implies that $R(\rho) \subset \pi_{\beta}\left(R\left(G_{\beta}\right)\right)$ for some $\beta \in \mathscr{D}$. Hence $\rho$ induces a rational representation $\rho_{\beta}$ of $G_{\beta}$ satisfying $\rho=\rho_{\beta} \circ h_{\beta} . \rho_{\beta}$ has a differential $d \rho_{\beta}$. We define the representation $d \rho$ of $\mathscr{L}$ by $d \rho=d \rho_{\beta} \circ d h_{\beta} . d \rho$ is clearly independent of the choice of $\beta$.

Now suppose that $(\rho, V)$ is locally finite. We define $d \rho: \mathscr{L} \rightarrow E(V)$ as follows: for each $l \in \mathscr{L}$ and $v \in V$, let $W$ be a finite-dimensional invariant subspace of $V$ containing $\rho(\mathscr{G})(v)$ and define $(d \rho)(l)$ by $(d \rho)(l)(v)=d\left(\rho_{w}\right)(l)(v)$. It is easily verified that $(d \rho)(l)(v)$ is independent of $W$. To show that $d \rho$ is a representation of $\mathscr{L}$, fix $l_{1}$ and $l_{2}$ in $\mathscr{L}$ and $v \in V$. Choose $W$ a finite dimensional invariant subspace of $V$ containing $v,(d \rho)\left(l_{1}\right)(v)$, and $(d \rho)\left(l_{2}\right)(v)$. Then

$$
\begin{aligned}
d \rho\left(\left[l_{1}, l_{2}\right]\right)(v) & =d\left(\rho_{w}\right)\left(\left[l_{1}, l_{2}\right]\right)(v)=\left[d\left(\rho_{w}\right)\left(l_{1}\right), d\left(\rho_{w}\right)\left(l_{2}\right)\right](v) \\
& =\left[d \rho\left(l_{1}\right), d \rho\left(l_{2}\right)\right](v) .
\end{aligned}
$$

Definition. $d \rho$ is called the differential of $\rho$.

It is easily verified that $d \rho$ is trivial if and only if $\rho$ is trivial, and that a subspace of $V$ is $\mathscr{G}$-invariant under $\rho$ if and only if it is $\mathscr{L}$-invariant under $d \rho$.

For each $\alpha \in \mathscr{D}$, denote by $S\left(L_{\alpha}\right)$ the algebra of representative functions on $L_{\alpha}$ which are associated with differentials of rational representations of $G_{\alpha}$. By analogy with the situation for the $R\left(G_{\alpha}\right)$, we may construct a direct limit algebra $S(\mathscr{L})$ of functions on $\mathscr{L}$. The following proposition follows easily.

Proposition 6.1. A locally finite representation $\rho$ of $\mathscr{L}$ is the differential of a rational representation of $\mathscr{G}$ if and only if the representative functions associated with $\rho$ lie in $S(\mathscr{L})$.

DefinITION. $\mathscr{G}$ is said to be irreducible if each $G_{\alpha}$ is an irreducible algebraic group.

Since $R(\mathscr{G})=\bigcup_{\alpha \in \mathscr{D}} \pi_{\alpha}\left(R\left(G_{\alpha}\right)\right)$, it is clear that $\mathscr{G}$ is irreducible if and only if $R(\mathscr{G})$ is an integral domain. 


\section{REFERENCES}

1. C. Chevalley, Théorie des groupes de Lie, Tome II, Paris, 1951.

2. G. Hochschild, Algebraic Lie algebras and representative functions, Illinois J. Math. 3 (1959), 499-523.

3. - Algebraic Lie algebras and representative functions. Supplement, Illinois J. Math 4 (1960), 609-618.

4. G. Hochschild and G. D. Mostow, Representations and representative functions of Lie groups, Ann. of Math. (2) 66 (1957), 495-542.

5. - On the algebra of representative functions of an analytic group, Amer. J. Math. 83 (1961), 111-136.

HARVARD UNIVERSITY,

Cambridge, Massachusetts 